\title{
Optimal inequalities for state-independent contextuality
}

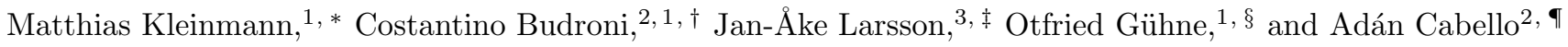 \\ ${ }^{1}$ Naturwissenschaftlich-Technische Fakultät, Universität Siegen, Walter-Flex-Straße 3, D-57068 Siegen, Germany \\ ${ }^{2}$ Departamento de Física Aplicada II, Universidad de Sevilla, E-41012 Sevilla, Spain \\ ${ }^{3}$ Institutionen för Systemteknik, Linköpings Universitet, SE-58183 Linköping, Sweden
}

\begin{abstract}
Contextuality is a natural generalization of nonlocality which does not need composite systems or spacelike separation and offers a wider spectrum of interesting phenomena. Most notably, in quantum mechanics there exist scenarios where the contextual behavior is independent of the quantum state. We show that the quest for an optimal inequality separating quantum from classical noncontextual correlations in an state-independent manner admits an exact solution, as it can be formulated as a linear program. We introduce the noncontextuality polytope as a generalization of the locality polytope, and apply our method to identify two different tight optimal inequalities for the most fundamental quantum scenario with state-independent contextuality.
\end{abstract}

PACS numbers: 03.65.Ta, 03.65.Ud

Introduction.- The investigation of the operational differences between quantum mechanics and classical mechanics resulted 1964 in the discovery of Bell's inequalities [1]. Such inequalities constrain the correlations obtained from spacelike-separated measurements and are satisfied by any local hidden variable (HV) model but are violated by quantum mechanics. For every measurement scenario, there exists a minimal set of inequalities, called tight Bell inequalities, which provide also sufficient conditions: If all tight inequalities are satisfied, then there exists a local HV model reproducing the corresponding set of correlations [2, 3].

Mathematically speaking, each tight Bell inequality corresponds to a facet of the locality polytope [3]. This means that it is an $(p-1)$-dimensional face of the $p$ dimensional polytope obtained as a convex hull of the vectors representing local assignments to the results of the considered measurements. Such a polytope gives all classical probabilities associated to a local model for a given measurement scenario, and its facets give precisely the boundaries of the polytope. In this sense, tight inequalities separate classical from nonclassical correlations perfectly.

Similarly, noncontextuality inequalities [4 6 ] are constraints on the correlations among the results of compatible observables, which are satisfied by any noncontextual $\mathrm{HV}$ model. While the violation of Bell inequalities reveals nonlocality, the violation of noncontextuality inequalities reveals contextuality [7, 8], which is a natural generalization of nonlocality privileging neither composite systems (among other physical systems), nor spacelike-separated measurements (among other compatible measurements), nor entangled states (among other quantum states).

All Bell inequalities are noncontextuality inequalities, but there are two features of noncontextuality inequalities which are absent in Bell inequalities. One is that noncontextuality inequalities may be violated by simple quantum systems such as single qutrits [4]. These violations have recently been experimentally observed with photons [9]. The other is that the violation can be independent of the quantum state of the systems [ $[5,[6]$, thus it reveals state-independent contextuality (SIC). The latter has been demonstrated recently with ququarts (four-level quantum systems) using ions [10], photons [11], and nuclear magnetic resonance [12].

The notion of tightness naturally applies also to noncontextuality inequalities. Tight noncontextuality inequalities are the facets of the correlation polytope of compatible observables as we will explain below. Compared with the locality polytope, the difference is in the notion of compatibility, since now one no longer considers only collections of spacelike-separated measurements, but admits more generally the measurement of a context, i.e., a collection of mutually compatible measurements. For a given contextuality scenario, the corresponding set of tight inequalities gives necessary and sufficient conditions for the existence of a noncontextual model.

For example, the three inequalities with stateindependent violation introduced in Ref. [5], are all tight. These inequalities are only violated for ququarts (two of the inequalities) and eight-level quantum systems (the third inequality), but not for qutrits. Another example of a tight inequality is the noncontextuality inequality for qutrits of Klyachko et al. [4], which indeed was derived by means of the correlation polytope method. However, this latter inequality does not have a state-independent quantum violation.

Obtaining all tight inequalities is, in general, a hard task. The correlation polytope is characterized by the number of settings and outcomes of the considered scenario. While there are algorithms that find all the facets of a given polytope, the time required to compute them grows exponentially as the number of settings increases. Therefore, this method can only be applied to simple cases with a reduced number of settings [2, 4, 13]. Given the facets of the polytope, in a next step one can try to find quantum observables that exhibit a maximal gap between the maximal noncontextual value and the quantum 
prediction.

In this paper we approach the problem differently. For many situations, the quantum observables are already known, and it remains to find inequalities that are tight and optimal and, in addition, may exhibit SIC. Thus we first describe the noncontextuality polytope for a given set of observables and a given list of admissible contexts. A noncontextuality inequality is then an affine hyperplane that does not intersect this polytope. We then introduce a method for maximizing the state-independent quantum violation via linear programming. The resulting linear program can be solved with standard optimization routines, and the optimality of the solution is guaranteed. As an application we derive the optimal inequality for several state independent scenarios, in particular analyzing a recently discovered qutrit scenario [14]. Using our method, we find noncontextuality inequalities with state-independent violation and the fewest number of observables and contexts. These inequalities turn out to be in addition tight and hence provide the most fundamental examples of inequalities with state-independent violation.

Contextuality scenarios, the noncontextuality polytope, and noncontextuality inequalities.-We start from some given dichotomic [15] quantum observables $A_{1}, A_{2}, \ldots, A_{n}$. A context $\underline{\mathfrak{c}}$ is then a set of indices, such that $A_{k}$ and $A_{\ell}$ are compatible whenever $k, \ell \in \mathfrak{c}$, i.e., $\left[A_{k}, A_{\ell}\right]=0$. For example if $A_{1}$ and $A_{2}$ are compatible, then valid contexts would be $\{1\},\{2\}$, and $\{1,2\}$. As we see below, it may be interesting to consider only a certain admissible subset $\mathfrak{C}$ of the set of all possible contexts $\{\underline{\mathfrak{c}}\}$. The observables $A_{1}, \ldots, A_{n}$, together with the list of admissible contexts $\mathfrak{C}$, form the contextuality scenario.

The set of all (contextual as well as noncontextual) correlations for such a scenario can be represented by the following standard construction. We first use that, if $A_{k}$ and $A_{\ell}$ are compatible, then the expectation value of $A_{k}$ is not changed whether or not $A_{\ell}$ is measured in the same context. Thus, instead of considering all correlations, it suffices to only consider the vector $\vec{v}=\left(v_{\underline{c}} \mid \underline{\mathfrak{c}} \in \mathfrak{C}\right)$, where $v_{\underline{c}}$ is the expectation value of the product of the values of the observables indexed by $\mathfrak{c}$. For example, for the contexts $\{1\},\{2\},\{1,2\}$, a contextual HV model may with equal probability assign the values $\{+1\},\{+1\}$, $\{-1,+1\}$, or $\{-1\},\{-1\},\{+1,-1\}$, respectively, yielding $\vec{v} \equiv\left(v_{1}, v_{2}, v_{1,2}\right)=(1 / 2,1 / 2,-1)$.

In the simplest noncontextual HV model, however, each observable has a fixed assignment $\vec{a} \equiv\left(a_{1}, \ldots, a_{n}\right) \in$ $\{-1,1\}^{n}$ for the observables $A_{1}, \ldots, A_{n}$, and accordingly each entry in $\vec{v}$ is exactly the product of the assigned values, i.e., $v_{\underline{c}}=\prod_{k \in \underline{\mathfrak{c}}} a_{k}$. The most general noncontextual HV model predicts fixed assignments $\vec{a}^{(i)}$ with probabilities $p_{i}$, and hence the set of correlations that can be explained by a noncontextual $\mathrm{HV}$ models is characterized by the convex hull of the models with fixed assignments, thus forming the noncontextuality polytope.

Then, a noncontextuality inequality is an affine bound on the noncontextuality polytope, i.e., a real vector $\vec{\lambda}$ such that $\eta \geq \vec{\lambda} \cdot \vec{v}$ for all correlation vectors $v$ that originate from a noncontextual model:

$$
\eta \geq \sum_{\underline{\mathfrak{c}} \in \mathfrak{C}} \lambda_{\underline{\mathfrak{c}}} \prod_{k \in \underline{\mathfrak{c}}} a_{k}
$$

for any assignment $\vec{a} \equiv\left(a_{1}, \ldots, a_{n}\right) \in\{-1,1\}^{n}$.

In quantum mechanics, in contrast, the measurement of the entry $v_{\underline{c}}$ corresponds to the expectation value $\left\langle\prod_{k \in \underline{\mathfrak{c}}} A_{k}\right\rangle_{\rho}$, where $\rho$ specifies the quantum state. Thus the value of $\vec{\lambda} \cdot \vec{v}$ predicted by quantum mechanics is given by $\langle T(\vec{\lambda})\rangle_{\rho}$, with

$$
T(\vec{\lambda})=\sum_{\underline{\mathfrak{c}} \in \mathfrak{C}} \lambda_{\underline{\mathfrak{c}}} \prod_{k \in \underline{\mathfrak{c}}} A_{k} .
$$

If the expectation value exceeds the noncontextual limit $\eta$, then the inequality demonstrates contextual behavior, yielding the quantum violation

$$
\mathcal{V}=\frac{\max _{\rho}\langle T(\vec{\lambda})\rangle_{\rho}}{\eta}-1
$$

An inequality is optimal, if the violation is maximal for the given contextuality scenario. In general, this optimization is difficult to perform and it is not always clear that an optimal inequality also yields the most significant violation [16].

Optimal state independent violation and tight inequalities.- However, if we require a state independent violation of the inequality, without loss of generality, $T(\vec{\lambda})=\mathbb{1}$ and hence the optimization over the quantum state $\varrho$ vanishes. Then, the coefficient vector $\vec{\lambda}$ and the noncontextuality bound $\eta$ are optimal if $\eta$ is minimal under the constraint $T(\vec{\lambda})=\mathbb{1}$ and if the inequalities in Eq. (1) are satisfied. That is, we ask for a solution $\left(\eta^{*}, \vec{\lambda}^{*}\right)$ of the optimization problem

$$
\begin{aligned}
& \text { minimize: } \eta \text {, } \\
& \text { subject to: } \quad T(\vec{\lambda})=\mathbb{1} \text { and } \\
& \text { Eq. (10) holds for all } \vec{a} \text {. }
\end{aligned}
$$

This optimization problem is a linear program and such programs can be solved efficiently by standard numerical techniques and optimality is then guaranteed. We implemented this optimization using CVXOPT [17] for Python, which allows us to study inequalities with up to $n=21$ observables and $|\mathfrak{C}|=131$ contexts. Note that this program also solves the feasibility problem, whether a contextuality scenario exhibits SIC at all. This is the case, if and only if the program finds a solution with $\eta<1$ and thus $\mathcal{V}>0$.

The optimal coefficients $\vec{\lambda}^{*}$ are, in general, not unique but rather form a polytope defined by Eq. (1) with $\eta=$ 


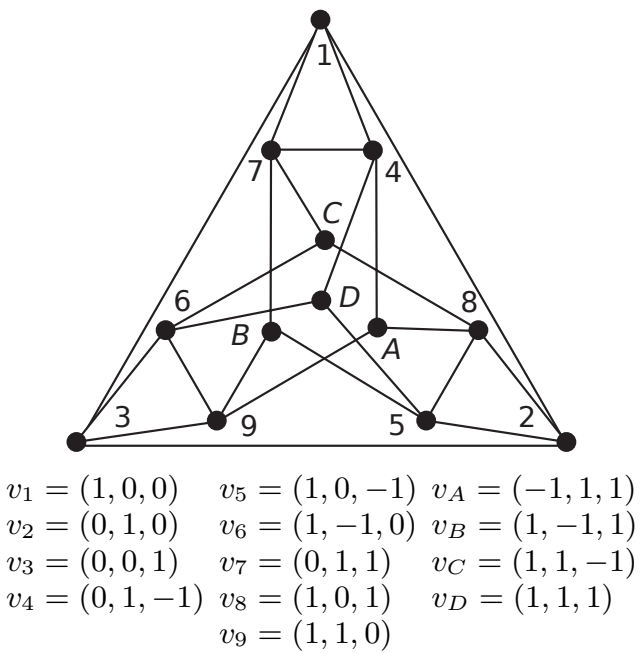

FIG. 1. Graph of the compatibility relations between the observables for the $\mathrm{Yu}-\mathrm{Oh}$ scenario. Nodes represent vectors $\left|v_{i}\right\rangle$ [or the observables $A_{i}$ defined in (5)] and edges represent orthogonality (or compatibility) relations.

$\eta^{*}$. This leaves the possibility to find optimal inequalities with further special properties. There are at least two important properties that one may ask for. Firstly, from an experimental point of view, it would be desirable to have some of the coefficients $\lambda_{\underline{\mathfrak{c}}}=0$, since then the context $\underline{\mathfrak{c}}$ does not need to be measured. In general, it will depend on the experimental setup, which coefficients $\lambda_{\underline{c}}=0$ yield the greatest advantage. For the sequential measurement schemes it is natural to choose the longest measurement sequences. Secondly, there might be tight inequalities among the optimal solutions: An inequality is tight, if the affine hyperplane given by the solutions of $\eta=\vec{\lambda} \cdot \vec{x}$ is tangent to a facet of the noncontextuality polytope. This property can be readily checked using Pitowsky's construction [3]: Denote by $p$ the affine dimension of the noncontextuality polytope and choose those assignments $\vec{a}$, for which Eq. (11) is saturated. Then, the inequality is tangent to a facet if and only if the affine space spanned by the vertices $\vec{v} \equiv\left(\prod_{k \in \mathfrak{c}} a_{k} \mid \underline{\mathfrak{c}} \in \mathfrak{C}\right)$ is $(p-1)$ dimensional.

Furthermore, we mention that the condition of state independence might be loosened to only require that the quantum violation is at least $\mathcal{V}$ for all quantum states. This corresponds to replacing the condition $T(\vec{\lambda})=\mathbb{1}$ by the condition that $T(\vec{\lambda})-\mathbb{1}$ is positive semidefinite. Then, the linear program in Eq. (4) becomes a semidefinite program, which still can be solved by standard numerical methods with optimality guaranteed. However, for the examples that we consider in the following, the semidefinite and the linear program yield the same results.

Most fundamental noncontextuality inequalities.-We now apply our method to the SIC scenario for a qutrit system introduced by $\mathrm{Yu}$ and $\mathrm{Oh}[14]$. Qutrit systems are

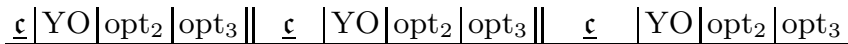

\begin{tabular}{r|c|c|c||r|r|r|r||r|r|r|r}
\hline 1 & 2 & 2 & 1 & $A D$ & 2 & 1 & 2 & 3,9 & -1 & -2 & -1 \\
2 & 2 & 3 & 1 & 1,2 & -1 & -1 & -2 & 4,7 & -1 & 0 & -1 \\
3 & 2 & 3 & 1 & 1,3 & -1 & -1 & -2 & 5,8 & -1 & -2 & -1 \\
4 & 2 & 1 & 1 & 1,4 & -1 & -1 & -1 & 6,9 & -1 & -2 & -1 \\
5 & 2 & 2 & 1 & 1,7 & -1 & -1 & -1 & $*, A D$ & -1 & -1 & -2 \\
6 & 2 & 2 & 1 & 2,3 & -1 & -2 & -2 & $1,2,3$ & - & - & 0 \\
7 & 2 & 1 & 1 & 2,5 & -1 & -2 & -1 & $1,4,7$ & - & - & -3 \\
8 & 2 & 2 & 1 & 2,8 & -1 & -2 & -1 & $2,5,8$ & - & - & -3 \\
9 & 2 & 2 & 1 & 3,6 & -1 & -2 & -1 & $3,6,9$ & - & - & -3
\end{tabular}

TABLE I. Coefficients $\lambda_{\underline{c}}$ of inequalities for the Yu-Oh scenario. The column $\underline{\mathfrak{c}}$ labels the different contexts, YO the coefficients in the inequality of Ref. 14], opt $\mathrm{ot}_{2}$ an optimal tight inequality with contexts of maximal size 2, opt 3 an optimal tight inequality with contexts of all sizes. For compactness, the coefficients in the column $\mathrm{YO}$ have been multiplied by $50 / 3$, for the column opt 2 by $52 / 3$ and for the column opt 3 by $83 / 3$. The row labeled " $A-D$ " shows the coefficients for the contexts $\{A\},\{B\},\{C\},\{D\}$ and the row labeled "*, $A$ $D$ " shows the coefficients for $\{4, A\},\{8, A\},\{9, A\},\{5, B\}$, $\{7, B\},\{9, B\},\{6, C\},\{7, C\},\{8, C\},\{4, D\},\{5, D\},\{6, D\}$.

of fundamental interest, since no smaller quantum system can exhibit a contextual behavior [8]. It has been shown, that this scenario is the simplest possible SIC scenario for a qutrit [18].

For a qutrit system, the dichotomic observables are of the form

$$
A_{i}=\mathbb{1}-2\left|v_{i}\right\rangle\left\langle v_{i}\right| .
$$

In the Yu-Oh scenario, there are 13 observables defined by the 13 unit vectors $\left|v_{i}\right\rangle$ provided in Fig. 1 In the according graph, each operator is represented by node $i \in V$ of the graph $G=(V, E)$ and an edge $(i, j) \in E$ indicates that $\left|v_{i}\right\rangle$ and $\left|v_{j}\right\rangle$ are orthogonal, $\left\langle v_{j} \mid v_{i}\right\rangle=0$, so that $A_{i}$ and $A_{j}$ are compatible. The original inequality takes into account all contexts of size one and two, $\mathfrak{C}_{\mathrm{YO}}=$ $\{\{1\}, \ldots,\{D\}\} \cup E$ and the coefficients were chosen to $\lambda_{\underline{\mathfrak{c}}}=-3 / 50$ if $\underline{\mathfrak{c}} \in E$ and $\lambda_{\underline{\mathfrak{c}}}=6 / 50$ else. This yields an inequality with a state-independent quantum violation of $\mathcal{V}=1 / 24 \approx 4.2 \%$.

With the linear program we find that the maximal violation for the contexts $\mathfrak{C}_{Y O}$ is $\mathcal{V}=1 / 12 \approx 8.3 \%$ and thus twice that of the inequality in Ref [14]. Interestingly, among the optimal coefficients $\vec{\lambda}^{*}$ there is a solution which is tight and for which the coefficients $\lambda_{4,7}$ vanishes, cf. Table [ column "opt 2 " for the list coefficients. We find that up to symmetries, $\lambda_{4,7}$ is the only context that can be omitted while still preserving optimality.

In order to demonstrate the practical advantage, let us discuss the recent experimental values obtained for the Yu-Oh scenario [19, FIG. 2]. For those values, the original $\mathrm{Yu}-\mathrm{Oh}$ inequality is violated by 3.7 standard deviations. But if the same data is evaluated using our optimal inequality "opt ${ }_{2}$ ", the violation increases to 7.5 
standard deviations. We mention, however, that the particular experimental setup implements the same observable in different context differently, thus easily allowing a noncontextual HV model explaining the data 20]. A setup avoiding such problems is described in Ref. [21].

The maximal contexts in the $\mathrm{Yu}-\mathrm{Oh}$ scenario are of size three, and hence it is possible to include also the corresponding terms in the inequality, i.e., we extend the contexts $\mathfrak{C}_{\text {YO }}$ by the contexts $\{1,2,3\},\{1,4,7\},\{2,5,8\}$, and $\{3,6,9\}$. Since this increases the number of parameters in the inequality, there is a chance that this case allows an even higher violation. In fact, the maximal violation is $\mathcal{V}=8 / 75 \approx 10.7 \%$. Again, it is possible to find tight inequalities with vanishing coefficients, and in particular the context $\{1,2,3\}$ can be omitted; the list of coefficients is given in Table I, column "opt 3 ".

Further examples.-Our method is applicable to all SIC scenarios, providing the optimal inequality. We mention two further examples: (i) The "extended PeresMermin square" uses as observables all 15 products of Pauli operators on a two-qubit system, $\left(\sigma_{\mu} \otimes \sigma_{\nu}\right)$ [22]. The optimal violation is $\mathcal{V}=2 / 3$, where only contexts of size three need to be measured and $\lambda_{\underline{c}}=1 / 15$, except $\lambda_{x x, y y, z z}=\lambda_{x z, y x, z y}=\lambda_{x y, y z, z x}=-1 / 15$. Among the optimal solutions no simpler inequality exists. (ii) The 18 vector proof [23] of the Kochen-Specker theorem uses a ququart system and 18 observables of the form (51). For contexts up to size 2 the maximal violation is $\mathcal{V}=1 / 17 \approx 5.9 \%$ (cf. 24 ), while including all context the maximal violation is $\mathcal{V}=2 / 7 \approx 28.6 \%$ (cf. [5]). The situation where only contexts up to size 3 are admissible has not yet been studied and we find numerically a maximal violation of $\mathcal{V} \approx 14.3 \%$.

Conclusions.-Contextuality is suspected to be one of the fundamental phenomena in quantum mechanics. While it can be seen as the underlying property of the nonlocal behavior of quantum mechanics, so far no methods for a systematic investigation have been developed. We here showed that Pitowsky's polytope naturally generalizes to the noncontextual scenario and hence the question of a full characterization of this noncontextuality polytope arises. This can be done via the so-called tight inequalities. On the other hand, among the most striking aspects where contextuality is more general than nonlocality is that the former can be found to be independent of the quantum state. For this state-independent scenario, we showed that the search for the optimal inequality reduces to a linear program, which can be solved numerically with optimality guaranteed. We studied several cases of this optimization and find that in all those instances one can construct noncontextuality inequalities with a state independent violation that are, in addition, tight. This is in particular the case for the most fundamental scenario of state independent contextuality 14] and we presented two essentially different inequalitiesone involves at most contexts of size two, the other of size three. We hence lifted the $\mathrm{Yu}-\mathrm{Oh}$ scenario to the same fundamental status as the $\mathrm{CHSH}$ Bell inequality 25], which is the simplest scenario for nonlocality. Our state-independent tight inequalities are particularly suitable for experimental tests and hence we expect that they stimulate experiments to finally observe SIC in qutrits [21].

The authors thank J. R. Portillo for checking some calculations. This work was supported by the Spanish Project No. FIS2011-29400, the EU (Marie-Curie CIG 293933/ENFOQI), the Austrian Science Fund (FWF): Y376-N16 (START prize), and the BMBF (CHIST-ERA network QUASAR).

* matthias.kleinmann@uni-siegen.de

† cbudroni@us.es

jan-ake.larsson@liu.se

$\S$ otfried.guehne@uni-siegen.de

a adan@us.es

[1] J. S. Bell, Physics 1, 195 (1964).

[2] A. Fine, Phys. Rev. Lett. 48, 291 (1982)

[3] I. Pitowsky, Quantum Probability-Quantum Logic (Springer, Berlin, 1982).

[4] A. A. Klyachko, M. A. Can, S. Binicioğlu, and A. S. Shumovsky, Phys. Rev. Lett. 101, 020403 (2008).

[5] A. Cabello, Phys. Rev. Lett. 101, 210401 (2008)

[6] P. Badziąg, I. Bengtsson, A. Cabello, and I. Pitowsky, Phys. Rev. Lett. 103, 050401 (2009).

[7] E. Specker, Dialectica 14, 239 (1960)

[8] J. S. Bell, Rev. Mod. Phys. 38, 447 (1966). S. Kochen and E. P. Specker, J. Math. Mech. 17, 59 (1967).

[9] R. Łapkiewicz, P. Li, C. Schaeff, N. K. Langford, S. Ramelow, M. Wieśniak, and A. Zeilinger, Nature (London) 474, 490 (2011); J. Ahrens, E. Amselem, A. Cabello, and M. Bourennane, (unpublished).

[10] G. Kirchmair, F. Zähringer, R. Gerritsma, M. Kleinmann, O. Gühne, A. Cabello, R. Blatt, and C. F. Roos, Nature (London) 460, 494 (2009).

[11] E. Amselem, M. Rådmark, M. Bourennane, and A. Cabello, Phys. Rev. Lett. 103, 160405 (2009).

[12] O. Moussa, C. A. Ryan, D. G. Cory, and R. Laflamme, Phys. Rev. Lett. 104, 160501 (2010).

[13] I. Pitowsky and K. Svozil, Phys. Rev. A 64, 014102 (2001); D. Collins and N. Gisin, J. Phys. A: Math. Gen. 37, 1775 (2004).

[14] S. Yu and C. H. Oh, Phys. Rev. Lett. 108, 030402 (2012).

[15] Dichotomy is not a restriction, since using the spectral decomposition $X=\sum \lambda_{i} \Pi_{i}$ the observable $X$ can be replaced by the dichotomic observables $X_{i}=2 \Pi_{i}-\mathbb{1}$.

[16] B. Jungnitsch, S. Niekamp, M. Kleinmann, O. Gühne, H. Lu, W.-B. Gao, Y.-A. Chen, Z.-B. Chen, and J.-W. Pan, Phys. Rev. Lett. 104, 210401 (2010).

[17] "CVXOPT," http://abel.ee.ucla.edu/cvxopt/.

[18] A. Cabello, arXiv:1112.5149v2.

[19] C. Zu, Y.-X. Wang, D.-L. Deng, X.-Y. Chang, K. Liu, P.Y. Hou, H.-X. Yang, and L.-M. Duan, Phys. Rev. Lett. 109, 150401 (2012).

[20] O. Gühne, M. Kleinmann, A. Cabello, J.-Å. Larsson, G. Kirchmair, F. Zähringer, R. Gerritsma, and C. F. 
Roos, Phys. Rev. A 81, 022121 (2010).

[21] A. Cabello, E. Amselem, K. Blanchfield, M. Bourennane, and I. Bengtsson, Phys. Rev. A 85, 032108 (2012)

[22] A. Cabello, Phys. Rev. A 82, 032110 (2010).

[23] A. Cabello, J. M. Estebaranz, and G. García-Alcaine, Phys. Lett. A 212, 183 (1996)
[24] S. Yu and C. Oh, arXiv:1112.5513v1.

[25] J. F. Clauser, M. A. Horne, A. Shimony, and R. A. Holt, Phys. Rev. Lett. 23, 880 (1969); Phys. Rev. Lett. 24, 549 (1970). 